\title{
Decreased Ratios of Interleukin-1 $\beta$ and Intercellular Adhesion Molecule 1 in Gingival Crevicular Fluid after Scaling and Root Planing Associated with Periodontal Pocket Healing
}

\author{
Yuriko Hieda, Michihiko Usui, Taiji Nakamura, Masaki Morishita, Tomoya Hanatani, \\ Yuki Moritani, Yukiko Koga, Shingo Kasai, Maki Inoue, Keisuke Nakashima* \\ Division of Periodontology, Department of Oral Functions, Kyushu Dental University, Kitakyushu, Japan \\ Email: *nakashimak@kyu-dent.ac.jp
}

How to cite this paper: Hieda, Y., Usui, M., Nakamura, T., Morishita, M., Hanatani, T., Moritani, Y., Koga, Y., Kasai, S., Inoue, M. and Nakashima, K. (2017) Decreased Ratios of Interleukin- $1 \beta$ and Intercellular Adhesion Molecule 1 in Gingival Crevicular Fluid after Scaling and Root Planing Associated with Periodontal Pocket Healing. Open Journal of Stomatology, 7, 361-376.

https://doi.org/10.4236/ojst.2017.79031

Received: July 27, 2017

Accepted: August 22, 2017

Published: August 25, 2017

Copyright ( 92017 by authors and Scientific Research Publishing Inc. This work is licensed under the Creative Commons Attribution International License (CC BY 4.0).

http://creativecommons.org/licenses/by/4.0/

\section{(c)}

\begin{abstract}
Objectives: Several researchers have investigated inflammatory cytokines in gingival crevicular fluid (GCF) from chronic periodontitis with clinical parameters after periodontal treatment. The aim of the present study was to identify the inflammatory cytokines present in GCF associated with periodontal pocket healing after scaling and root planing (SRP). Methods: Twenty patients were enrolled, and clinical examinations including probing pocket depth (PPD) and bleeding on probing (BOP) were performed at the first visit, and before and after SRP. The periodontal epithelial surface area (PESA) and periodontal inflamed surface area (PISA) were also calculated. GCF samples were collected before and after SRP to measure the levels of 40 inflammatory cytokines using antibody array. Correlations between changes in cytokine levels and clinical improvements were assessed by single and multiple regression analyses. Results: PPD, PESA and PISA significantly decreased after SRP, while there were no significant differences in the levels of cytokines before (PreSRP) and one week after SRP (PostSRP). Clinical improvements ( $\triangle P P D$, $\triangle$ PESA and $\triangle$ PISA) were calculated using the formula PreSRP-PostSRP, while changes in the levels of inflammatory cytokines were calculated using three formulas: PreSRP-PostSRP, PostSRP/PreSRP and Log(PostSRP/PreSRP). Log (PostSRP/PreSRP) for interleukin-1 $\beta$ (IL- $1 \beta$ ) significantly correlated with $\triangle$ PESA $(\mathrm{R}=-0.512)$. PostSRP/PreSRP and Log(PostSRP/PreSRP) for intercellular adhesion molecule 1 (ICAM-1) significantly correlated with $\triangle \mathrm{PISA}(\mathrm{R}$ $=-0.669,-0.573)$. Two good fit models were generated by multiple linear regression. $\log \left(\right.$ PostSRP/PreSRP) for IL- $1 \beta$ significantly affected $\triangle \mathrm{PESA}\left(\mathrm{R}^{2}=\right.$ 0.566). PostSRP/PreSRP for ICAM-1 significantly affected $\triangle P I S A\left(R^{2}=0.615\right)$.
\end{abstract}


Conclusion: Changes (PreSRP-PostSRP) in any cytokines in GCF after SRP were not associated with clinical improvements. However, the present study indicates that lower PostSRP/PreSRP ratios for IL- $1 \beta$ and ICAM- 1 in GCF lead to a greater decrease in PESA and PISA, respectively. Periodontal pocket healing might be affected by a decreased ratio of inflammatory cytokines such as IL- $1 \beta$ and ICAM- 1 at an early stage after SRP.

\section{Keywords}

IL-1 $\beta$, ICAM-1, GCF, SRP, Wound Healing

\section{Introduction}

Cytokines are low molecular weight glycoprotein biomarkers that are involved in cellular interaction [1] [2]. Cytokines are secreted by hematopoietic cells, such as macrophages, and nonhematopoietic cells, such as epithelial cells, in response to infection by a microorganism. Inflammatory cytokines, which are induced during the inflammatory response, have been associated with the onset or progression of tissue destruction [3]. Thus, inflammatory cytokines are suggested to have a pleiotropic effect, targeting specific cells by controlling cellular activation.

Periodontitis is a chronic inflammatory response, which is caused by periodontopathic microorganisms in dental biofilm, and results in the progressive destruction of periodontal tissue [4] [5] [6]. The trigger of the inflammatory process in periodontitis is thought to be an increased secretion of inflammatory cytokines such as interleukin (IL)- $1 \alpha$, IL- $1 \beta$, tumor necrosis factor (TNF)- $\alpha$ and IL-6 [7] [8]. Thereafter, neutrophils, macrophages, fibroblasts and epithelial cells release destructive enzymes such as matrix metalloproteinases (MMPs), inflammatory mediators such as IL- $1 \beta$ and osteoclast-activating factor, and receptor activator of nuclear factor kappa-B ligand (RANKL) in response to inflammatory cytokines [9] [10]. These biochemicals play an essential role in extracellular matrix degradation and osteoclast differentiation, which results in collagen and alveolar bone destruction in periodontal tissue [11].

Gingival crevicular fluid (GCF) is an inflammatory exudate or plasma transudate, which can be non-invasively collected from the gingival sulcus surrounding the teeth [12] [13]. Several researchers have shown that GCF from patients with periodontitis contains inflammatory cytokines, such as IL-1, IL-6, TNF- $\alpha$ and IL-8, and destructive factors, such as MMPs and RANKL, the concentrations of which are significantly increased when compared with healthy samples [14]. Thus, it is suggested that GCF analysis may be useful for diagnosing the current periodontal condition and evaluating the effect of periodontal treatment. However, the amount of GCF collected from periodontal pockets is very small; therefore, GCF analysis must focus on a limited number of cytokines [15]. Recently, Sakai et al. investigated the expression of 42 cytokines in GCF from both healthy and periodontitis patients using a cytokine array system [16]. Although 
they identified the expression of 36 cytokines in periodontal pockets and $10 \mathrm{cy}-$ tokines in healthy sites, their research design was cross-sectional and the difference in age between the healthy and periodontitis patients was significant. Furthermore, the therapeutic effect markers in periodontitis are not yet fully understood.

The treatment of periodontitis aims for a reduction in supra- and subgingival biofilm, which contains periodontopathic microorganisms. This is mainly accomplished by mechanical removal of the bacterial deposits from the tooth surface by scaling and root planing (SRP). However, decreases in probing pocket depth (PPD) after SRP vary for individual patients, even when periodontal treatment is performed in the same way by the same periodontist. In other words, although SRP reduces PPD in patients with chronic periodontitis, some periodontal pockets do not react to SRP treatment and the anaerobic environment persists. We hypothesized that analyzing the GCF profile from periodontitis patients could enable assessment of the therapeutic response to periodontal treatment such as SRP. Periodontal wound healing after SRP consists of several phases. Twenty-four hours after SRP, a widespread infiltration of inflammatory cells and migration of keratinocytes have been observed. The entire surface area of the periodontal pocket is epithelialized in 2 days. In 4 - 5 days, a new epithelial attachment appears at the base of the sulcus. Depending on the severity of the inflammation and the depth of the gingival crevice, complete epithelial healing occurs in one week. Then, connective tissue repair by collagen fibers occurs with the formation of a long junctional epithelium within 21 days [17]. We assumed that the biological reaction after epithelialization of the gingival sulcus following SRP may relate to the healing of periodontal tissues in periodontitis. In the present study, we performed cytokine expression profiling in GCF before and one week after SRP using an inflammatory cytokine array kit and investigated the association of the initial changes in inflammatory cytokine levels with decreases in PPD to identify a pattern of cytokine expression that represented healing of periodontal tissue.

\section{Materials and Methods}

\subsection{Patient Selection and Experimental Protocol}

A total of 20 patients were selected from new patients with chronic localized periodontitis, who visited Kyushu Dental University Hospital from 2013 to 2015 (Table 1). Periodontitis was assessed by clinical examination, with classifications determined according to criteria proposed by the 1999 International World Workshop for a Classification of Periodontal Diseases and Conditions [18]. The enrollment criteria were as follows:

1) Patients who had received no periodontal treatment within 5 years

2) Patients who had a periodontal pocket $\geq 4 \mathrm{~mm}$ in depth in the incisor, canine or premolar region

3) Patients with at least 20 teeth 
Table 1. Patient profiles at the first visit.

\begin{tabular}{|c|c|c|c|c|c|c|c|}
\hline $\begin{array}{l}\text { Subject } \\
\text { number }\end{array}$ & Age & Gender & $\begin{array}{l}\text { Tooth } \\
\text { number }\end{array}$ & $\begin{array}{l}\text { Sample } \\
\text { site }\end{array}$ & $\begin{array}{l}\text { PPD } \\
(\mathrm{mm})\end{array}$ & $\begin{array}{l}\text { BOP } \\
(+/-)\end{array}$ & $\begin{array}{c}\text { Bone loss } \\
(\%)\end{array}$ \\
\hline 1 & 49 & Male & 32 & DB & 5 & + & 13 \\
\hline 2 & 57 & Male & 32 & DL & 7 & + & 10 \\
\hline 3 & 65 & Male & 25 & DB & 5 & - & 21 \\
\hline 4 & 62 & Male & 21 & $\mathrm{MB}$ & 7 & - & 63 \\
\hline 5 & 56 & Male & 13 & DB & 6 & + & 27 \\
\hline 6 & 49 & Female & 45 & $\mathrm{DL}$ & 5 & + & 34 \\
\hline 7 & 68 & Male & 25 & DB & 6 & + & 26 \\
\hline 8 & 74 & Female & 24 & DB & 4 & - & 35 \\
\hline 9 & 68 & Female & 32 & DB & 5 & + & 26 \\
\hline 10 & 60 & Female & 15 & DB & 4 & - & 20 \\
\hline 11 & 60 & Female & 24 & $\mathrm{MB}$ & 5 & - & 26 \\
\hline 12 & 66 & Female & 23 & ML & 6 & + & 38 \\
\hline 13 & 62 & Male & 42 & DB & 5 & - & 40 \\
\hline 14 & 53 & Male & 15 & DL & 4 & - & 29 \\
\hline 15 & 74 & Female & 12 & DB & 7 & + & 83 \\
\hline 16 & 69 & Female & 21 & $\mathrm{DL}$ & 6 & + & 26 \\
\hline 17 & 69 & Female & 21 & DB & 6 & - & 29 \\
\hline 18 & 65 & Female & 34 & $\mathrm{DL}$ & 7 & + & 28 \\
\hline 19 & 70 & Female & 25 & $\mathrm{MB}$ & 6 & + & 60 \\
\hline 20 & 63 & Female & 11 & $\mathrm{DL}$ & 5 & - & 21 \\
\hline
\end{tabular}

Periodontal probing was performed by qualified periodontists at the first visit. PPD: Probing pocket depth, BOP: Bleeding on probing, Bone loss (\%) was derived from the following formula: $100 \times \mathrm{B} / \mathrm{A}$; A: Distance between the cementoenamel junction and root apex; B: Distance between the cementoenamel junction and the base of the bone defect.

\section{4) Patients who were systemically healthy}

For each patient, a comprehensive clinical examination of the entire dentition including periodontal probing, oral hygiene assessment and dental radiographs was performed at the first visit (Figure 1(a)). PPD and bleeding on probing (BOP) were measured by qualified periodontists using a periodontal probe ( $\# 2$, YDM, Tokyo, Japan) at six sites per tooth. A sample site in the incisor, canine or premolar region at which the PPD was $\geq 4 \mathrm{~mm}$ was then determined for each patient. The distance between the cementoenamel junction and root apex (A) and the distance between the cementoenamel junction and the base of the bone defect (B) were measured on dental radiographs. Bone loss (\%) was derived from the following formula: $100 \times \mathrm{B} / \mathrm{A}$ [19].

Thereafter, all patients received oral hygiene instruction, which was repeated several times. Periodontal probing was performed after a high standard of oral hygiene was achieved (PreSRP PPD). At the next visit, GCF was collected from the sample 
(a) Clinical examination (PPD, BOP, X-ray, PCR) on a first visit to determine a sample site for each patient

।

Oral hygiene instruction for several times

I

Clinical examination (PreSRP PPD)

I

GCF collection from the sample site (PreSRP GCF) and SRP performed using ultrasonic scaler and specific Gracey curettes

। GCF collection from the sample site at 7 days after SRP (PostSRP GCF)

। Clinical examination (PostSRP PPD) after the initial periodontal treatment had been completed (approximately after 2 to 3 months)

(b)

\begin{tabular}{|c|c|c|c|c|c|c|c|c|c|c|c|}
\hline POS & POS & NEG & NEG & Eotaxin & Eotaxin-2 & G-CSF & GM-CSF & ICAM-1 & IFN-y & I-309 & IL-1a \\
\hline POS & POS & NEG & NEG & Eotaxin & Eotaxin-2 & G-CSF & GM-CSF & ICAM-1 & IFN-y & 1-309 & $\mathrm{IL}-1 \alpha$ \\
\hline IL-1 $\beta$ & IL-2 & IL-3 & IL-4 & IL-6 & IL-6 sR & IL-7 & IL-8 & IL-10 & IL-11 & IL-12-p40 & IL-12 p70 \\
\hline IL-1 $\beta$ & IL-2 & IL-3 & IL-4 & IL-6 & IL-6 sR & IL-7 & IL-8 & IL-10 & IL-11 & IL-12-p40 & IL-12 p70 \\
\hline IL-13 & IL-15 & IL-16 & IL-17 & IP-10 & MCP-1 & MCP-2 & $\mathrm{M}-\mathrm{CSF}$ & MIG & MIP-1a & MIP-1 $\beta$ & MIP-1ס \\
\hline IL-13 & IL-15 & IL-16 & IL-17 & IP-10 & MCP-1 & MCP-2 & $\mathrm{M}-\mathrm{CSF}$ & MIG & MIP-1a & MIP-1 $\beta$ & MIP-1ס \\
\hline RANTES & TGF- $\beta 1$ & TNF- $\alpha$ & TNF- $\beta$ & sTNF-RI & sTNF RII & PDGF-BB & TIMP-2 & BLANK & BLANK & NEG & POS \\
\hline RANTES & TGF- $\beta 1$ & TNF- $\alpha$ & TNF- $\beta$ & sTNF-RI & sTNF RII & PDGF-BB & TIMP-2 & BLANK & BLANK & NEG & POS \\
\hline
\end{tabular}

Figure 1. Research protocol. (a) Research protocol, (b) Cytokines detectable with human cytokine antibody array C3, POS: Positive control, NEG: Negative control.

site of each patient just prior to SRP (PreSRP GCF), and then collected from the same site one week after SRP (PostSRP GCF). SRP was performed by several operators using an ultrasonic scaler (EMS Electro Medical Systems S.A., Nyon, Switzerland) and specific Gracey curettes (Hu-Friedy Mfg. Co., LLC, Chicago, IL, USA). All patients received quadrant SRP at 2-3 weeks intervals. Periodontal probing was performed after the initial periodontal treatment had been completed (PostSRP PPD). The periodontal epithelial surface area (PESA) and periodontal inflamed surface area (PISA) were also calculated as described by Nesse et al. [20].

This research protocol was approved by the ethics committee of Kyushu Dental University (\#12-10). All patients received a detailed explanation regarding the study protocol, and written informed consent was obtained from those who agreed to participate in this study. The present study was conducted in accordance with the Helsinki Declaration.

\subsection{GCF Collection}

GCF was carefully collected from the sample site of each patient. Saliva and pla- 
que were removed from the site of GCF collection isolated with cotton wool rolls. A paper point (\#40, Morita Co., Tokyo, Japan) was gently inserted into the sample site and left in place for 30 seconds. The paper point was transferred into $120 \mu \mathrm{l}$ of ice-cold sterilized phosphate-buffered saline and stored at $-80^{\circ} \mathrm{C}$ until analysis with a cytokine antibody array. Any GCF samples with bleeding were excluded. The GCF was thawed at room temperature immediately before the assay. After vortexing for 30 seconds and centrifuging at $800 \times g$ for 1 minute, the paper points were removed. The supernatants were used as GCF samples.

\subsection{Cytokine Antibody Array}

Semi-quantitative, sandwich-based, membrane antibody array (Human Inflammation Array C3; RayBiotech, Inc., Atlanta, GA, USA) was used to simultaneously detect 40 cytokines according to the manufacturer's instructions (Figure $1(b))$. Briefly, the cytokine assay membrane wells were blocked with 2 ml blocking buffer at room temperature for 30 minutes, and were then incubated with the samples diluted to $1 \mathrm{ml}$ at $4^{\circ} \mathrm{C}$ overnight. After aspirating the samples, the membranes were washed with wash buffers I and II, and incubated with biotinylated antibody cocktail at $4^{\circ} \mathrm{C}$ overnight. The membranes were washed with wash buffers again and incubated with streptavidin-conjugated horseradish peroxidase $(1: 1000)$ at $4^{\circ} \mathrm{C}$ overnight. After washing out unbound reagents with wash buffers, the membranes were incubated in detection buffer for 2 minutes. Exposure started within 5 minutes after incubation, and the chemiluminescent signal from the bound cytokine was detected on GelDoc ${ }^{\mathrm{Tm}}$ XR Plus (Bio-Rad, San Francisco, CA, USA). Intensities of spots of cytokines from scanned images were calculated using Image J (NIH, Baltimore, MD, USA). Finally, these data were analyzed using the RayBio ${ }^{\circledR}$ antibody array analysis tool specifically designed for the arrays (RayBiotech, Inc.). Data for spots with lower intensities than the background could not be calculated and were handled as missing data in the statistical analysis.

\subsection{Statistical Analysis}

Statistical analyses were performed using the JMP 12.2.0 software program (SAS Institute Inc., Cary, NC, USA).

\section{Results}

The patients that participated in the present study consisted of eight males and twelve females with an average age of $62.8 \pm 7.0$ years (Table 1). PPD at sample sites ranged from $4-7 \mathrm{~mm}$. We first compared the clinical measurements (PreSRP PPD and PostSRP PPD) before and after SRP. Distributions of PPDs were shown as a box-and-whisker plot with seven percentile lines $(0,10,25,50$, $75,90,100)$ as the data were not fit to a normal distribution (Figure 2). Matched pairs analysis using the Wilcoxon signed-rank test revealed that PPD significantly decreased after SRP $(p<0.001)$. Wilcoxon signed-rank test also revealed 

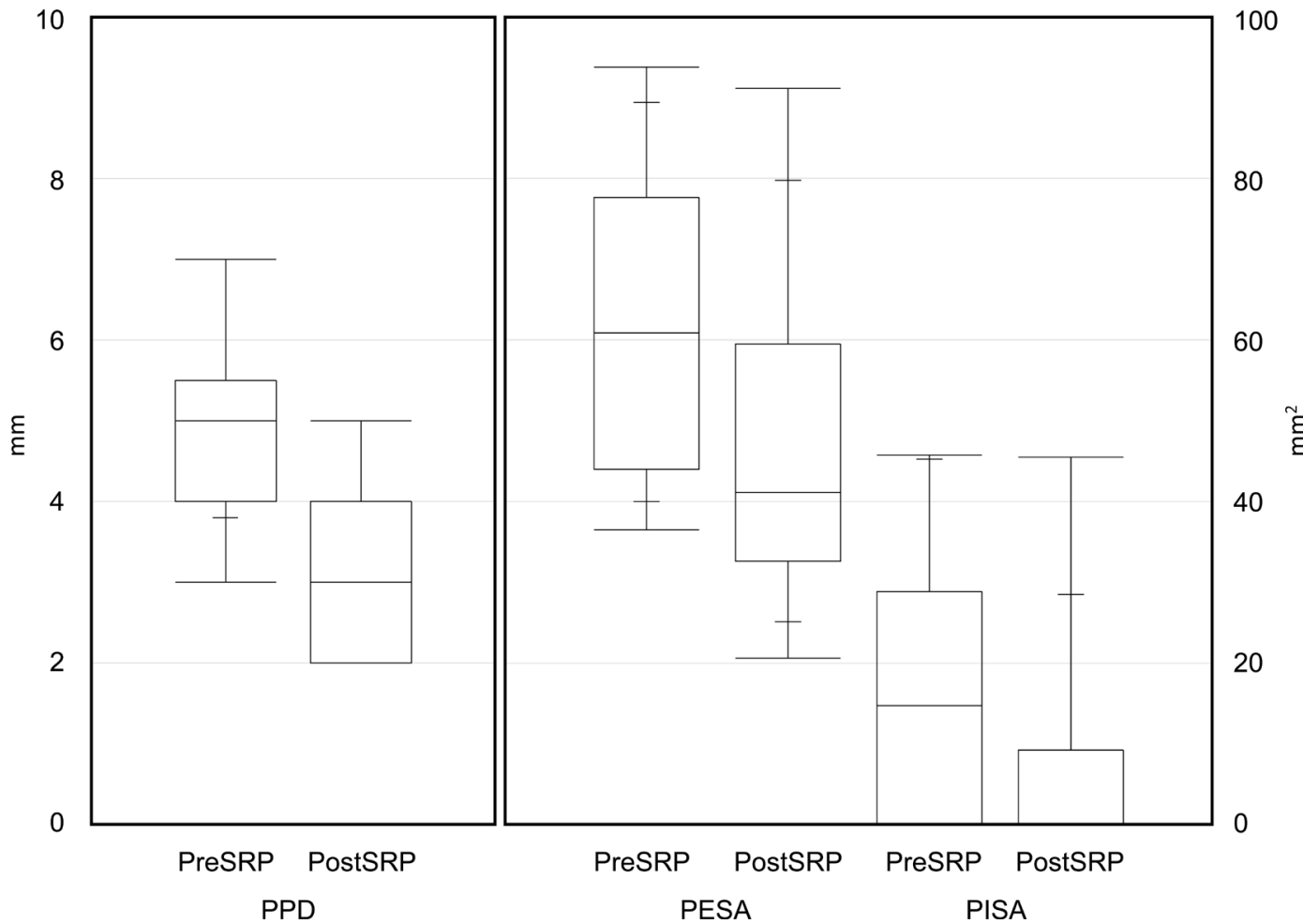

Figure 2. Distribution of PPD, PESA and PISA before and after SRP. Periodontal probing was performed by qualified periodontists before SRP (PreSRP) and after initial periodontal treatment had been completed (PostSRP). Time intervals were 2 to 3 months between periodontal probing before and after SRP. Periodontal epithelial surface area (PESA) and periodontal inflamed surface area (PISA) were calculated using probing pocket depth (PPD) and bleeding on probing (BOP) according to reference [19]. Distributions of PPD, PESA and PISA were shown as a box-and-whisker plot with seven percentile lines $(0,10,25,50,75$, $90,100)$. Wilcoxon signed-rank test revealed that PPD, PESA and PISA significantly decreased after SRP ( $p$ $<0.001, p<0.001$ and $p=0.032$ ).

that PESA and PISA significantly decreased after SRP $(p<0.001$ and $p=0.032)$.

We then compared cytokine levels in GCF samples collected before and one week after SRP (Figure 3). The number of samples detected for each cytokine varied from eight to seventeen, as no visible spot was recognized on the membranes for some cytokines; therefore, this was handled as missing data. Distributions of levels of cytokines in GCF before and after SRP were shown as box-and-whisker plots with seven percentile lines $(0,10,25,50,75,90,100)$, as the data were not fit to a normal distribution. The median for IL-8 levels in GCF was 1.4-20-fold higher when compared with the other cytokines. However, matched pairs analysis using the Wilcoxon signed-rank test revealed no significant difference between the levels of any cytokines in GCF before and after SRP.

Finally, we investigated the association of changes in cytokine levels in GCF one week after SRP with clinical improvements 2 to 3 months after SRP. Clinical improvements ( $\triangle \mathrm{PPD}, \triangle \mathrm{PESA}$ and $\triangle \mathrm{PISA}$ ) for PPD, PESA and PISA were calculated using the formula PreSRP-PostSRP. Changes in cytokine levels in GCF were then calculated using the following three formulas: PreSRP-PostSRP, PostSRP/PreSRP and Log (PostSRP/PreSRP). The changes in cytokine levels in 


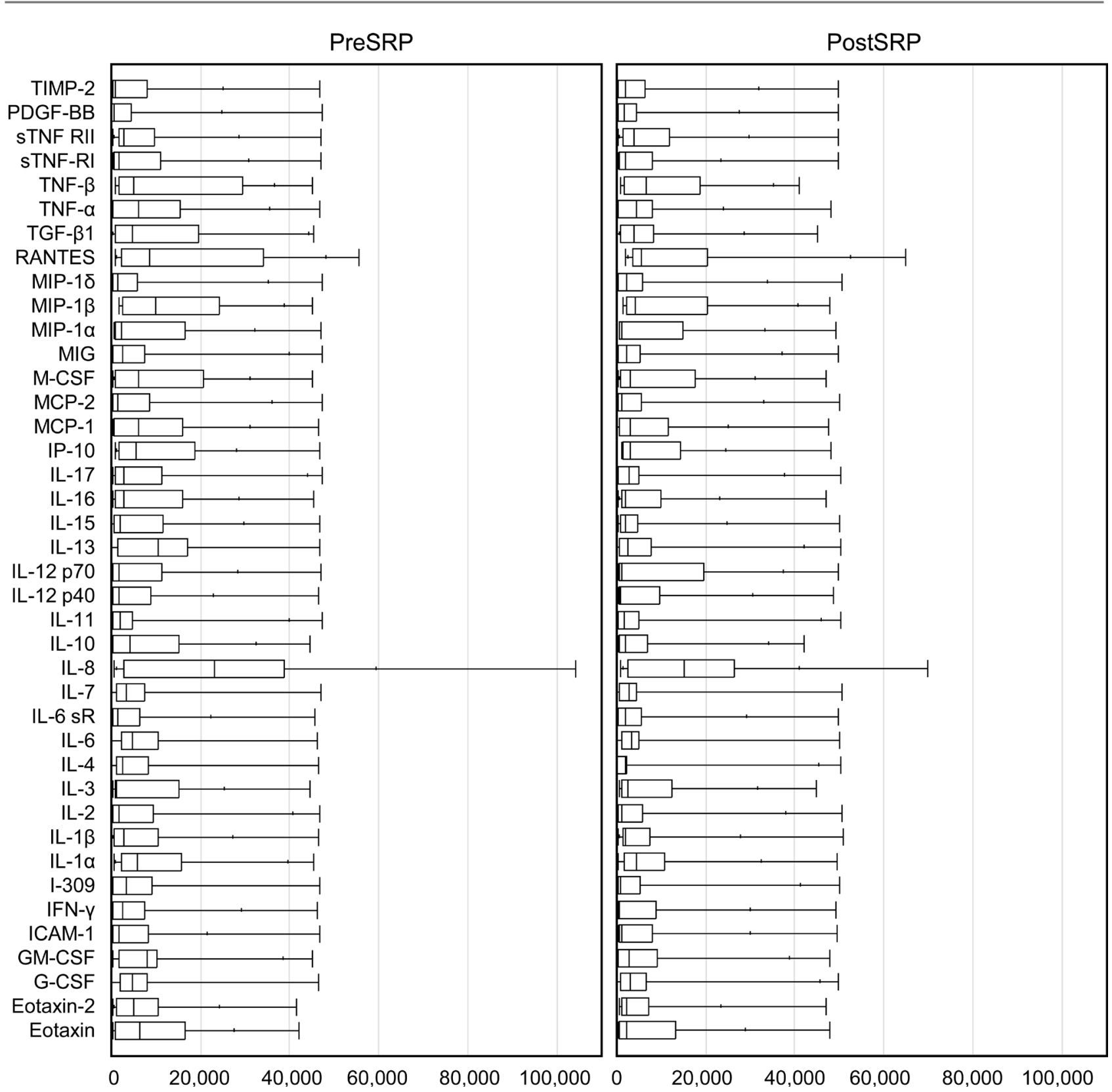

Figure 3. Distributions of levels of cytokines before and after SRP. GCF samples were collected before (PreSRP) and 7 days after SRP (PostSRP). Distributions of levels of cytokines in GCF were shown as a box-and-whisker plot with seven percentile lines (0, $10,25,50,75,90,100)$. The levels of any cytokines in GCF did not significantly change after SRP (Wilcoxon signed-rank test).

GCF calculated using the formula PreSRP-PostSRP mostly ranged from $-40,000$ to 40,000 , while changes calculated using the formula $\log ($ PostSRP/PreSRP) mostly ranged from -3 to 3 (Figure 4 ). Correlations between clinical improvements ( $\triangle \mathrm{PPD}, \triangle \mathrm{PESA}$ and $\triangle \mathrm{PISA}$ ) and changes in cytokine levels in GCF calculated using the three formulas, PreSRP-PostSRP, PostSRP/PreSRP and Log (PostSRP/PreSRP), were examined (Table 2). There were no significant correlations except $\triangle$ PESA vs. $\log ($ PostSRP/PreSRP) for IL-1 $\beta$ (correlation coefficient $=-0.512, p=0.043$ ), $\Delta$ PISA vs. PostSRP/PreSRP for ICAM-1 (correlation coefficient $=-0.669, p=0.009)$ and $\Delta$ PISA vs. $\log ($ PostSRP/PreSRP $)$ for ICAM-1 
(correlation coefficient $=-0.573, p=0.049$ ).

Two good fit models were generated by multiple linear regression. Log (PostSRP/PreSRP) for IL- $1 \beta$ significantly affected $\triangle \mathrm{PESA}\left(\mathrm{R}^{2}=0.566\right.$, adjusted $\mathrm{R}^{2}=0.421, p=0.049$ ) (Table 3 ). PostSRP/PreSRP for ICAM-1 significantly affected $\Delta$ PISA $\left(\mathrm{R}^{2}=0.615\right.$, adjusted $\left.\mathrm{R}^{2}=0.480, p=0.029\right)($ Table 4$)$.

\section{Discussion}

In the present study, we investigated the levels of inflammatory cytokines in

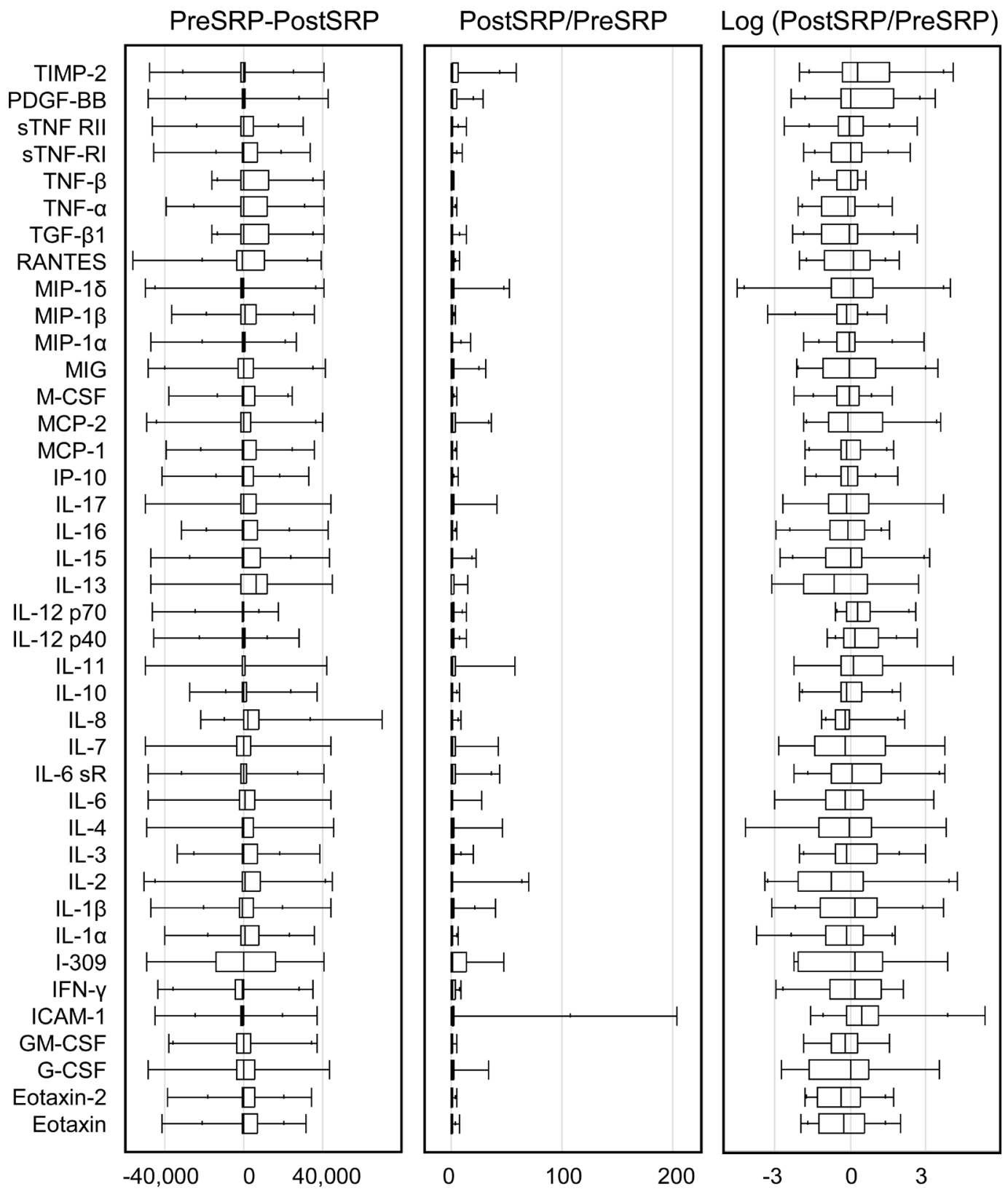

Figure 4. Distributions of changes in the levels of cytokines after SRP. Changes in the levels of cytokines in GCF were calculated using three formulas: PreSRP-PostSRP, PostSRP/PreSRP and Log (PostSRP/PreSRP). Distributions of changes in levels of cytokines were shown as a box-and-whisker plot with seven percentile lines $(0,10,25,50,75,90,100)$. 
Table 2. Correlation between clinical improvements ( $\triangle \mathrm{PPD}, \triangle \mathrm{PESA}$ and $\triangle \mathrm{PISA})$ and changes in cytokine levels.

\begin{tabular}{rcccc}
\hline & & PreSRP-PostSRP & PostSRP/PreSRP & $\log ($ PostSRP/PreSRP) \\
\hline \multicolumn{1}{c}{ PPD } & & & & \\
& ICAM-1 & -0.015 & -0.254 & -0.253 \\
& IL-1 $\beta$ & 0.072 & -0.300 & -0.405 \\
$\Delta$ PESA & & & & -0.359 \\
& ICAM-1 & 0.141 & -0.383 & $-0.512^{*}$ \\
& IL-1 $\beta$ & 0.206 & -0.441 & \\
\multirow{2}{*}{$\Delta$ PISA } & & & & $-0.573^{*}$ \\
& ICAM-1 & -0.063 & $-0.669^{*}$ & -0.242 \\
\hline
\end{tabular}

Correlation coefficients are shown in this table. ${ }^{\star} p<0.05$. PPD: Probing pocket depth, PESA: Periodontal epithelial surface area, PISA: Periodontal inflamed surface area Clinical improvements $(\triangle \mathrm{PPD}, \triangle \mathrm{PESA}$ and $\triangle$ PISA) were calculated using the formula PreSRP-PostSRP. Changes in cytokine levels in GCF were calculated using the following three formulas: PreSRP-PostSRP, PostSRP/PreSRP and Log (PostSRP/PreSRP). There were no significant correlations between clinical improvements and changes in cytokine levels except $\triangle$ PESA vs. Log (PostSRP/PreSRP) for IL-1 $\beta, \triangle$ PISA vs. PostSRP/PreSRP for ICAM-1 and $\Delta$ PISA vs. Log (PostSRP/PreSRP) for ICAM-1.

Table 3. $\triangle$ PESA estimation formula derived by performing a multiple linear regression Log (PostSRP/PreSRP).

\begin{tabular}{ccccc}
\hline Term & Estimate & Std Error & t Ratio & Prob $>|t|$ \\
\hline Intercept & 14.014 & 2.839 & 4.935 & 0.001 \\
IL-1 $\beta$ & -13.226 & 4.159 & -3.180 & 0.011 \\
MCP-1 & 17.641 & 8.814 & 2.001 & 0.076 \\
TNF- $\beta$ & 0.543 & 7.516 & 0.072 & 0.944 \\
\hline
\end{tabular}

$\triangle$ PESA and changes in cytokine level calculated using the formula Log (PostSRP/PreSRP) were used as objective and explanatory variables, respectively. $\mathrm{R}^{2}=0.566$, adjusted $\mathrm{R}^{2}=-0.421, p=0.049$.

Table 4. $\triangle$ PISA estimation formula derived by performing a multiple linear regression PostSRP/PreSRP.

\begin{tabular}{ccccc}
\hline Term & Estimate & Std Error & t Ratio & Prob $>|t|$ \\
\hline Intercept & 13.473 & 5.127 & 2.628 & 0.027 \\
ICAM-1 & -0.181 & 0.072 & -2.531 & 0.032 \\
IL-10 & -2.115 & 1.994 & -1.061 & 0.317 \\
sTNF RII & 1.625 & 1.033 & 1.573 & 0.150 \\
\hline
\end{tabular}

$\triangle \mathrm{PISA}$ and changes in cytokine level calculated using the formula PostSRP/PreSRP were used as objective and explanatory variables, respectively. $\mathrm{R}^{2}=0.615$, adjusted $\mathrm{R}^{2}=-0.486, p=0.029$.

GCF from patients with chronic periodontitis using the human inflammation antibody array kit, which enabled the semi-quantitative detection of 40 inflammatory cytokines simultaneously. We demonstrated that clinical measurements (PPD, PESA and PISA) significantly improved after SRP, although the levels of 
40 inflammatory cytokines in GCF did not significantly change after SRP. The results of the present study, however, indicate that a lower PostSRP/PreSRP ratio for IL- $1 \beta$ and ICAM- 1 in GCF led to a greater decrease in PESA and PISA, respectively. This is the first comprehensive analysis of inflammatory cytokines in GCF during the early phase of periodontal healing after SRP associated with clinical improvement.

Presently, periodontitis is well-known to be an inflammatory condition of the supporting tissues of teeth caused by periodontopathic microorganisms, which induce the host immune and inflammatory responses [21]. Therefore, it is believed that the intensity of periodontal inflammation could be assessed objectively by analyzing GCF components, because many inflammatory and immune mediators implicated in periodontal destruction have been detected in GCF. Several researchers have reported that the expression of inflammatory cytokines such as IL-1, IL-6, IL- 8 and TNF- $\alpha$ in GCF from chronic periodontitis patients was significantly elevated when compared with healthy patients [22] [23] [24] [25]. Furthermore, there are some reports investigating the effect of nonsurgical periodontal therapy involving SRP on GCF from patients with chronic periodontitis [26] [27]. Hannigan et al. reported a significant decrease in soluble ICAM-1 in GCF from patients with chronic periodontitis after SRP [28]. Konopka et al. found a marked decrease in IL- $1 \beta$ in GCF at 4 weeks after SRP along with a significant improvement in clinical parameters [23]. At one week after SRP, GCF samples from five patients showed almost the same levels of IL- $1 \beta$ as the baseline, while samples from the other 25 patients showed lower levels than the baseline. All the samples from 30 patients eventually showed significantly lower levels of IL- $1 \beta$ than the baseline at 4 weeks. They failed to find any significant correlations between the amounts of IL- $1 \beta$, IL-8 and MMP-8 in GCF and clinical parameters at 1 and 4 weeks after SRP. It might be noted that there were two patterns of decreases of IL- $1 \beta$ in GCF after SRP. Our present study showed that the levels of IL- $1 \beta$ in GCF did not significantly decrease by one week after SRP (Figure 3). Furthermore, there was no expression of IL- $1 \beta$ in three out of 20 samples. The range of IL- $1 \beta$ levels, which could be detected in 17 GCF samples, were also very broad (median: 2652, range: 38 - 46,444) (Figure 3). The detection limit of the human inflammation antibody array kit, which we used in this study, was $100 \mathrm{pg} / \mathrm{ml}$, while the IL- $1 \beta$ enzyme-linked immunosorbent assay (ELISA) kit used by Konopka et al. could detect $2 \mathrm{pg} / \mathrm{ml}$. Therefore, the difference in results between these studies may be explained by the detection limit of the antibody array kit used.

Antibody array kits have several advantages when compared with single protein target ELISA kits. One of the advantages of antibody arrays is the ability to perform high-content screening using approximately the same volume as traditional ELISA. We detected EOTAXIN-2, I-309, monocyte chemotactic protein (MCP)-2, monokine induced by interferon-gamma, and macrophage inflammatory protein (MIP)- $1 \delta$, which, to our knowledge, have not been previously detected in GCF. EOTAXIN-2, I-309, MCP-2 and MIP- $1 \delta$ are known as chemo- 
kine (C-C motif) ligands CCL24, CCL1, CCL8 and CCL15, respectively, and belong to the CC chemokine family. These chemokines have been reported to be chemotactic and to activate several immune cells [29] [30] [31] [32]. Although SRP did not affect the expression of these novel cytokines in GCF, they may be involved in the pathogenesis of periodontitis. Further analyses are needed to prove this hypothesis. Conversely, antibody array also has some disadvantages, such as the narrow range of detection on target proteins as described above. In addition, antibody array is less accurate in detecting the coefficient of variation of spot signal intensities of proteins as determined by densitometry, when compared with conventional ELISA methods. Improvement of these disadvantages would make the antibody array a useful tool for analysis of GCF.

In our study, we found that there was no significant correlation between the clinical parameters and the expression of 40 inflammatory cytokines in GCF after SRP therapy. Several researchers have investigated the relationship between clinical parameters and the levels of inflammatory cytokines such as IL- $1 \beta$ and IL-8 after SRP. Toker et al. found that there were no significant correlations between the level of IL- $1 \beta$ and clinical parameters 6 weeks after initial periodontal therapy [33]. Research by Gamonal et al. also showed a very weak correlation between clinical parameters and the levels of IL- $1 \beta$ and IL- 82 months after SRP [34]. These data suggest that it is difficult to explain the periodontal tissue condition using the levels of inflammatory cytokines alone. In the present study, we hypothesized that normal levels of cytokines vary for each individual patient; therefore, we evaluated changes in cytokine levels using PostSRP/PreSRP in addition to PreSRP-PostSRP. We revealed that $\log ($ PostSRP/PreSRP) for IL-1 $\beta$ was significantly correlated with a decrease in PESA ( $\triangle \mathrm{PESA})$ in Table 2. PostSRP/PreSRP and Log(PostSRP/PreSRP) for ICAM-1 were also significantly correlated with a decrease in PISA ( $\triangle \mathrm{PISA})$. Moreover, multiple linear regression indicates that a lower ratio of PostSRP/PreSRP for IL- $1 \beta$ and ICAM-1 in GCF leads to a greater decrease in PESA and PISA. We could not specify the cytokines that affect the decrease in PPD after SRP. This may be because only small changes are seen after SRP in sites with moderate PPD.

GCF contains host-derived enzymes, such as MMPs, and growth factors, such as platelet-derived growth factor- $\mathrm{BB}$, which are important regulators of the healing process. Thus, there may be a correlation between clinical parameters and multiple factors, including inflammatory cytokines, enzymes and growth factors. Furthermore, we used additional indexes, PESA and PISA, which were calculated using six PPD sites and BOP scores obtained per target tooth using the six-point probing method. Previous studies related to GCF generally examined the PPD or clinical attachment level at the single deepest pocket per tooth, and used this value as a clinical parameter. We inferred that GCF in a periodontal pocket of one tooth would be affected by other pockets around the same tooth because the gingival sulcus exists continuously around the tooth. In fact, our single and multiple regression analyses indicated that the change in the expression of inflammatory cytokines correlated with $\triangle \mathrm{PESA}$ and $\triangle \mathrm{PISA}$, not 
with $\triangle \mathrm{PPD}$. Although several researchers reported no correlation between the change in levels of inflammatory cytokines and clinical parameters such as PPD after SRP [34], statistical analysis with the addition of $\triangle \mathrm{PESA}$ and $\triangle \mathrm{PISA}$ as a clinical parameter may have shown a correlation.

Numerous researchers have demonstrated the beneficial effects of SRP on both clinical and microbial parameters [35]. Our observation also indicated significant improvement in clinical parameters after initial periodontal treatment. However, the expression of inflammatory cytokines was still elevated one week after SRP. Therefore, the findings of this research may suggest that nonsurgical treatment, such as SRP, results in improved clinical signs of inflammation, and that the wound healing process is affected by the decreased ratio of inflammatory cytokines such as IL- $1 \beta$ and ICAM- 1 at an early stage after SRP. Periodontal healing is a highly complex process under the control of inflammatory cytokines, MMPs and growth factors. More detailed investigation is needed to identify the healing process of periodontal tissue.

\section{Acknowledgements}

This research was supported in part by Grants-in-Aid from the Japan Society for the Promotion of Science, Kakenhi (grant numbers 24593129 and 15K11399), to $\mathrm{KN}$. We are grateful to the past and present members of our division. We thank Angie Smaranda, M (Dent), from Edanz Group (http://www.edanzediting.com/ac) for editing a draft of this manuscript. The authors report no conflicts of interest related to this study.

\section{References}

[1] Hanada, T. and Yoshimura, A. (2002) Regulation of Cytokine Signaling and Inflammation. Cytokine \& Growth Factor Reviews, 13, 413-421. https://doi.org/10.1016/S1359-6101(02)00026-6

[2] Seymour, G.J. and Gemmell, E. (2001) Cytokines in Periodontal Disease: Where to from Here? Acta Odontologica Scandinavica, 59, 167-173. https://doi.org/10.1080/000163501750266765

[3] Gemmell, E., Marshall, R.I. and Seymour, G.J. (1997) Cytokines and Prostaglandins in Immune Homeostasis and Tissue Destruction in Periodontal Disease. Periodontology 2000, 14, 112-143. https://doi.org/10.1111/j.1600-0757.1997.tb00194.x

[4] Bostanci, N. and Bao, K. (2017) Contribution of Proteomics to Our Understanding of Periodontal Inflammation. Proteomics, Vol. 17.

https://doi.org/10.1002/pmic.201500518

[5] Graves, D.T. (1999) The Potential Role of Chemokines and Inflammatory Cytokines in Periodontal Disease Progression. Clinical Infectious Diseases, 28, 482-490. https://doi.org/10.1086/515178

[6] Heilbronn, L.K. and Campbell, L.V. (2008) Adipose Tissue Macrophages, Low Grade Inflammation and Insulin Resistance in Human Obesity. Current Pharmaceutical Design, 14, 1225-1230. https://doi.org/10.2174/138161208784246153

[7] De Lima Oliveira, A.P., de Faveri, M., Gursky, L.C., Mestnik, M.J., Feres, M., Haffajee, A.D., Socransky, S.S. and Teles, R.P. (2012) Effects of Periodontal Therapy on 
GCF Cytokines in Generalized Aggressive Periodontitis Subjects. Journal of Clinical Periodontology, 39, 295-302. https://doi.org/10.1111/j.1600-051X.2011.01817.x

[8] Graves, D. (2008) Cytokines That Promote Periodontal Tissue Destruction. Journal of Periodontology, 79, 1585-1591. https://doi.org/10.1902/jop.2008.080183

[9] Kinney, J.S., Morelli, T., Oh, M., Braun, T.M., Ramseier, C.A., Sugai, J.V. and Giannobile, W.V. (2014) Crevicular Fluid Biomarkers and Periodontal Disease Progression. Journal of Clinical Periodontology, 41, 113-120. https://doi.org/10.1111/jcpe.12194

[10] Ngo, L.H., Darby, I.B., Veith, P.D., Locke, A.G. and Reynolds, E.C. (2013) Mass Spectrometric Analysis of Gingival Crevicular Fluid Biomarkers Can Predict Periodontal Disease Progression. Journal of Periodontal Research, 48, 331-341. https://doi.org/10.1111/jre.12012

[11] Fujihara, R., Usui, M., Yamamoto, G., Nishii, K., Tsukamoto, Y., Okamatsu, Y., Sato, T., Asou, Y., Nakashima, K. and Yamamoto, M. (2014) Tumor Necrosis Factor-Alpha Enhances RANKL Expression in Gingival Epithelial Cells via Protein Kinase a Signaling. Journal of Periodontal Research, 49, 508-517. https://doi.org/10.1111/jre.12131

[12] AlRowis, R., AlMoharib, H.S., AlMubarak, A., Bhaskardoss, J., Preethanath, R.S. and Anil, S. (2014) Oral Fluid-Based Biomarkers in Periodontal Disease Part 2. Gingival Crevicular Fluid. Journal of International Oral Health, 6, 126-135.

[13] Baliban, R.C., Sakellari, D., Li, Z., DiMaggio, P.A., Garcia, B.A. and Floudas, C.A. (2012) Novel Protein Identification Methods for Biomarker Discovery via a Proteomic Analysis of Periodontally Healthy and Diseased Gingival Crevicular Fluid Samples. Journal of Clinical Periodontology, 39, 203-212.

https://doi.org/10.1111/j.1600-051X.2011.01805.x

[14] Becerik, S., Ozturk, V.O., Atmaca, H., Atilla, G. and Emingil, G. (2012) Gingival Crevicular Fluid and Plasma Acute-Phase Cytokine Levels in Different Periodontal Diseases. Journal of Periodontology, 83, 1304-1313. https://doi.org/10.1902/jop.2012.110616

[15] Shimada, Y., Tabeta, K., Sugita, N. and Yoshie, H. (2013) Profiling Biomarkers in Gingival Crevicular Fluid Using Multiplex Bead Immunoassay. Archives of Oral Biology, 58, 724-730.

[16] Sakai, A., Ohshima, M., Sugano, N., Otsuka, K. and Ito, K. (2006) Profiling the Cytokines in Gingival Crevicular Fluid Using a Cytokine Antibody Array. Journal of Periodontology, 77, 856-864. https://doi.org/10.1902/jop.2006.050340

[17] Stanton, G., Levy, M. and Stahl, S.S. (1969) Collagen Restoration in Healing Human Gingiva. Journal of Dental Research, 48, 27-31. https://doi.org/10.1177/00220345690480011901

[18] Armitage, G.C. (1999) Development of a Classification System for Periodontal Diseases and Conditions. Annals of Periodontology, 4, 1-6. https://doi.org/10.1902/annals.1999.4.1.1

[19] Lira-Junior, R., Freires Ide, A., de Oliveira, I.L., da Silva, E.S., da Silva, S. and de Brito, R.L. (2013) Comparative Study between Two Techniques for Alveolar Bone Loss Assessment: A Pilot Study. Journal of Indian Society of Periodontology, 17, 87-90. https://doi.org/10.4103/0972-124X.107481

[20] Nesse, W., Abbas, F., van der Ploeg, I., Spijkervet, F.K., Dijkstra, P.U. and Vissink, A. (2008) Periodontal Inflamed Surface Area: Quantifying Inflammatory Burden. Journal of Clinical Periodontology, 35, 668-673. https://doi.org/10.1111/j.1600-051X.2008.01249.x 
[21] Armitage, G.C. (2004) Periodontal Diagnoses and Classification of Periodontal Diseases. Periodontology 2000, 34, 9-21. https://doi.org/10.1046/j.0906-6713.2002.003421.x

[22] De Oliveira, R.R., Schwartz-Filho, H.O., Novaes, A.B., Garlet, G.P., de Souza, R.F., Taba, M., Scombatti de Souza, S.L. and Ribeiro, F.J. (2009) Antimicrobial Photodynamic Therapy in the Non-Surgical Treatment of Aggressive Periodontitis: Cytokine Profile in Gingival Crevicular Fluid Preliminary Results. Journal of Periodontology, 80, 98-105. https://doi.org/10.1902/jop.2009.070465

[23] Konopka, L., Pietrzak, A. and Brzezinska-Blaszczyk, E. (2012) Effect of Scaling and Root Planing on Interleukin-1 Beta, Interleukin-8 and MMP-8 Levels in Gingival Crevicular Fluid from Chronic Periodontitis Patients. Journal of Periodontal Research, 47, 681-688. https://doi.org/10.1111/j.1600-0765.2012.01480.x

[24] Kurtis, B., Tuter, G., Serdar, M., Akdemir, P., Uygur, C., Firatli, E. and Bal, B. (2005) Gingival Crevicular Fluid Levels of Monocyte Chemoattractant Protein-1 and Tumor Necrosis Factor-Alpha in Patients with Chronic and Aggressive Periodontitis. Journal of Periodontology, 76, 1849-1855.

https://doi.org/10.1902/jop.2005.76.11.1849

[25] Tymkiw, K.D., Thunell, D.H., Johnson, G.K., Joly, S., Burnell, K.K., Cavanaugh, J.E., Brogden, K.A. and Guthmiller, J.M. (2011) Influence of Smoking on Gingival Crevicular Fluid Cytokines in Severe Chronic Periodontitis. Journal of Clinical Periodontology, 38, 219-228. https://doi.org/10.1111/j.1600-051X.2010.01684.X

[26] Thunell, D.H., Tymkiw, K.D., Johnson, G.K., Joly, S., Burnell, K.K., Cavanaugh, J.E., Brogden, K.A. and Guthmiller, J.M. (2010) A Multiplex Immunoassay Demonstrates Reductions in Gingival Crevicular Fluid Cytokines Following Initial Periodontal Therapy. Journal of Periodontal Research, 45, 148-152. https://doi.org/10.1111/j.1600-0765.2009.01204.x

[27] Yoshinari, N., Kawase, H., Mitani, A., Ito, M., Sugiishi, S., Matsuoka, M., Shirozu, N., Ishihara, Y., Bito, B., Hiraga, M., Arakawa, K. and Noguchi, T. (2004) Effects of Scaling and Root Planing on the Amounts of Interleukin-1 and Interleukin-1 Receptor Antagonist and the mRNA Expression of Interleukin-1 Beta in Gingival Crevicular Fluid and Gingival Tissues. Journal of Periodontal Research, 39, 158-167. https://doi.org/10.1111/j.1600-0765.2004.00722.x

[28] Hannigan, E., O’Connell, D.P., Hannigan, A. and Buckley, L.A. (2004) Soluble Cell Adhesion Molecules in Gingival Crevicular Fluid in Periodontal Health and Disease. Journal of Periodontology, 75, 546-550. https://doi.org/10.1902/jop.2004.75.4.546

[29] Elmoselhi, H., Mansell, H., Soliman, M. and Shoker, A. (2016) Circulating Chemokine Ligand Levels before and after Successful Kidney Transplantation. Journal of Inflammation, 13, 32. https://doi.org/10.1186/s12950-016-0141-4

[30] Forssmann, U., Uguccioni, M., Loetscher, P., Dahinden, C.A., Langen, H., Thelen, M. and Baggiolini, M. (1997) Eotaxin-2, a Novel CC Chemokine That Is Selective for the Chemokine Receptor CCR3, and Acts like Eotaxin on Human Eosinophil and Basophil Leukocytes. The Journal of Experimental Medicine, 185, 2171-2176. https://doi.org/10.1084/jem.185.12.2171

[31] Hellier, S., Frodsham, A.J., Hennig, B.J., Klenerman, P., Knapp, S., Ramaley, P., Satsangi, J., Wright, M., Zhang, L., Thomas, H.C., Thursz, M. and Hill, A.V. (2003) Association of Genetic Variants of the Chemokine Receptor CCR5 and Its Ligands, RANTES and MCP-2, with Outcome of HCV Infection. Hepatology, 38, 1468-1476.

[32] Miller, M.D. and Krangel, M.S. (1992) The Human Cytokine I-309 Is a Monocyte 
Chemoattractant. Proceedings of the National Academy of Sciences, 89, 2950-2954. https://doi.org/10.1073/pnas.89.7.2950

[33] Toker, H., Poyraz, O. and Eren, K. (2008) Effect of Periodontal Treatment on IL-1 Beta, IL-1ra, and IL-10 Levels in Gingival Crevicular Fluid in Patients with Aggressive Periodontitis. Journal of Clinical Periodontology, 35, 507-513. https://doi.org/10.1111/j.1600-051X.2008.01213.x

[34] Gamonal, J., Acevedo, A., Bascones, A., Jorge, O. and Silva, A. (2000) Levels of Interleukin-1 Beta, -8 , and -10 and RANTES in Gingival Crevicular Fluid and Cell Populations in Adult Periodontitis Patients and the Effect of Periodontal Treatment. Journal of Periodontology, 71, 1535-1545.

https://doi.org/10.1902/jop.2000.71.10.1535

[35] Rosalem, W., Rescala, B., Teles, R.P., Fischer, R.G., Gustafsson, A. and Figueredo, C.M. (2011) Effect of Non-Surgical Treatment on Chronic and Aggressive Periodontitis: Clinical, Immunologic, and Microbiologic Findings. Journal of Periodontology, 82, 979-989. https://doi.org/10.1902/jop.2011.100579

\section{Submit or recommend next manuscript to SCIRP and we will provide best} service for you:

Accepting pre-submission inquiries through Email, Facebook, LinkedIn, Twitter, etc. A wide selection of journals (inclusive of 9 subjects, more than 200 journals)

Providing 24-hour high-quality service

User-friendly online submission system

Fair and swift peer-review system

Efficient typesetting and proofreading procedure

Display of the result of downloads and visits, as well as the number of cited articles

Maximum dissemination of your research work

Submit your manuscript at: http://papersubmission.scirp.org/

Or contact ojst@scirp.org 